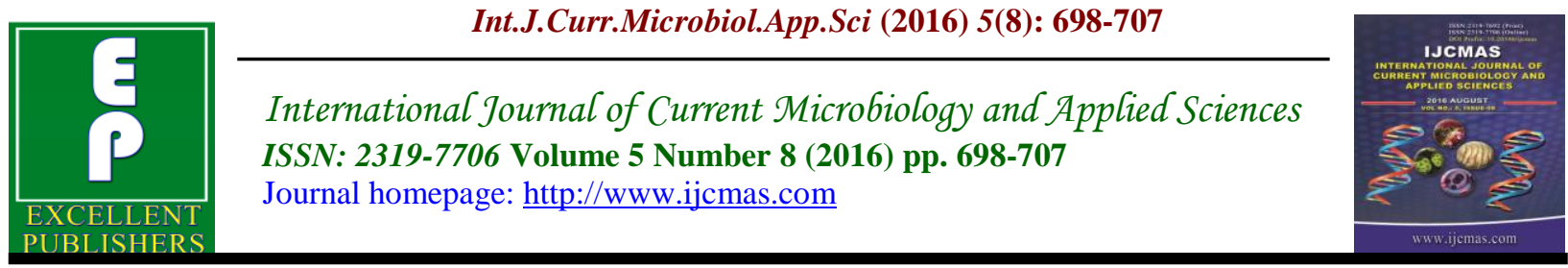

Original Research Article

http://dx.doi.org/10.20546/ijcmas.2016.508.079

\title{
A Study of Seroprevalence of Hepatitis B, Hepatitis C and Syphilis Coinfection among HIV Patients in a Tertiary Care Teaching Hospital, South India
}

\author{
Konda Palli Harathi Reddy, Uma Penmetcha*, Ramesh Babu Myneni, \\ and Padmaja Yarlagadda \\ Department of Microbiology, NRI Medical College and General Hospital, \\ Chinakakani, Mangalagiri Mandal, Guntur District, Andhra Pradesh, India \\ *Corresponding author
}

\section{Keywords}

HIV,

Co-infections,

Syphilis,

Hepatitis B,

Hepatitis C.

\section{Article Info}

Accepted:

28 July 2016

Available Online:

10 August 2016

\section{A B S T R A C T}

Human Immunodeficiency virus (HIV) with co infection of hepatitis B virus (HBV), Hepatitis $\mathrm{C}$ virus (HCV) and syphilis have the potential to increase the morbidity and mortality. The study was undertaken to find out the Seroprevalence of HIV in suspected individual for the years 2009 - 2010 \& to randomly selected every fourth HIV positive serum sample as material for further study, to assess the age \& gender of the HIV positive persons, to find out the route of transmission / acquisition of HIV in the subjects, to identify the category of high risk groups that the HIV positive individuals fall into, to determine the seroprevalence of co-infection of $\mathrm{HBV}, \mathrm{HCV}$ and VDRL in HIV positive subjects. This prospective study included an analysis of limited serological markers for Hepatitis B and C viruses, syphilis in HIV positive individuals. The total number of blood samples received for detection of HIV antibodies was 17105 during one year, of which 417 samples were reactive for either HIV- I only or HIV I \& II. The HIV positive rate was $2.43 \%$. The study was conducted on 104 serum samples reactive for HIV. Every $4^{\text {th }}$ HIV reactive sample was included in the study. Therefore the total number of HIV positive samples included in the study was 104. Age wise distribution of HIV positive patients whose samples were included in the study showed that $54.8 \%$ belong to the age group $30-44$ years and $26.9 \%$ belongs to 45 and above age group. Males accounted for $60.5 \%$ while $39.4 \%$ were females. The male to female ratio being 1.5:1. The commonest route of HIV transmission in our study was found to be sexual (73.07\%). Individuals with various STDS 52\%, CSW's and unknown formed the majority of the high risk groups, Unknown $23.07 \%$, CSW $20.19 \%$ respectively. Men having sex with men accounted for $2.88 \%$ of the total. Seroprevalence of three STD's in the study group was HbsAg 5(4.8\%), HCV antibodies $2(1.9 \%)$, VDRL reactive cases $7(6.7 \%)$. In our study no combined association of Hepatitis marker and syphilis in the HIV positive cases were found. None of the individual showed a dual or combined presence of all 3 markers (HBV + HCV + VDRL positivity). The present study showed that HIV infected individuals have a increased risk of getting coinfection with either Syphilis, HBV and / or HCV. Sexual transmission of both HBV and HCV also appears to be significant. Hence, timely treatment of these infections in HIV infected individual will decrease the complications associated with hepatitis viruses as well as the morbidity and mortality. 


\section{Introduction}

Human Immunodeficiency virus (HIV) with co infection of hepatitis B virus (HBV), Hepatitis $\mathrm{C}$ virus (HCV) and syphilis have the potential to increase the morbidity and mortality. Syphilis presenting with genital ulcerative lesions serve greater chance of transmission of HIV. Patients presenting with syphilis should be tested for HIV and vice versa. The main mode of spread of HIV, HBV and HCV in India is heterosexual, with blood products and intravenous drug use. Detection of HBV and HCV co infection in patients with HIV/AIDs is most important due to the underlying complications such as the liver disorders associated with these viruses, which has shown to decrease the life expectancy in the HIV infected patients (Chung et al., 2006). Among the HIV infected patients, 2.4 millions are estimated to have chronic HBV co infection. HIV infection enhances the progression of $\mathrm{HCV}$ related liver disease. With this background the AIMS and OBJECTIVES of the study was to find out the Seroprevalence of HIV in suspected individual for the years $2009-2010$ \& to randomly select every fourth HIV positive serum sample as material for further study, to assess the age \& gender of the HIV positive persons, whose serum samples formed the study material, to find out the route of transmission / acquisition of HIV in the subjects, to identify the category of high risk groups that the HIV positive individuals fall into, to determine the individual seroprevalence HBV, HCV and VDRL positivity in HIV positive subjects, to detect dual association of hepatitis markers \& marker of syphilis in the subjects as follows
A) HBV marker \& VDRL positivity
B) HCV marker \& VDRL positivity
C) HBV marker \& HCV positivity

To determine a combined presence of three markers namely -

A) $\mathrm{HBV}$ marker + $\mathrm{HCV}$ marker + VDRL positivity and to compare our results with those of other workers.

\section{Materials and Methods}

This prospective study was undertaken in the department of microbiology, NRI medical college \& Hospital, Chinakakani over a period of one year, from May 2009 to May 2010. During the one year study period, 17105 blood samples were received after pretest counseling and informed consent taken from patients attending the various clinical departments of our institution based on clinical suspicion, for HIV testing. Out of 17105 blood samples subjected to HIV testing, 417 serum samples were reactive for HIV. A random selection was then made by including every $4^{\text {th }}$ sample from the HIV reactive samples for further study. Thus, 104 serum samples formed the study material. 104 seropositive individuals selected for the study were then subjected to the following questionnaire:

Age \& gender; Possible route of transmission / acquisition of HIV infection; Detail history was undertaken, to determine the probable category of high risk group that each individual could be assigned to.

All the serum samples were then subjected to serological tests to determine the presence of limited markers for Hepatitis B, Hepatitis $C$ viruses \& Syphilis. These included the following:

a. ELISA test for surface antigen (HbsAg) of Hepatitis B

b. ELISA test for antibodies to $\mathrm{HCV}$

c. VDRL test for syphilis. 


\section{HIV Testing}

The samples were first tested with one ELISA or rapid or simple assay with higher sensitivity. Reactive serum samples were retested with a second ELISA with high specificity, based on a different antigen preparation \& /or different test principles as per NACO guidelines.

All ELISA \& rapid test procedures were followed strictly as per the instruction manual provided with each kit. ELISA test results were read by measuring the absorbance of each sample \& as controls on ELISA reader using the appropriate filters as recommended in the kit manual.

Calculation \& interpretation of result was done according to the kit manual, with the "cut off" \& "test validity" criteria being strictly adhered to. Any sample that was found to be positive by two tests was considered reactive.

\section{Viral Diagnosis}

The serum samples which were found to be HIV positive were stored at -20 degree Celsius after appropriate labelling. The labelled samples were further tested using enzyme linked immunosorbent assay kits at a later date for the presence of HBsAg (Hepanostika HBsAg Ultra, BioMerieux, Netherlands), anti HCV antibodies (Hepanostika HCV Ultra, Beijing united Biomedical Co., Ltd. Beijing), the test used for diagnosis of syphilis was a Rapid plasma reagin (RPR) card test which employs detection of reagenic antilipoidal antibodies. (Carbogen, Tulip, diagnostic (Pvt LTD) Goa, India).

All reactive samples with a VDRL titre of more than or equal to 1: 8 were considered as a positive test result to obviate biological false positive results.

\section{Results and Discussion}

This prospective study, extending from May 2009 to 2010, included an analysis of limited serological markers for Hepatitis B, Hepatitis C viruses and Syphilis in HIV positive individuals. The samples were received for HIV testing, from suspected individuals, attending the various Clinical Departments of this hospital. The total number of blood samples received for detection of HIV antibodies; which were to the tune of 17105 . The number of samples received in 2009 was 11139 , while 5966 samples were tested in 2010. The HIV positivity rate in 2009 was $2.7 \%$ while in 2010 it was $1.5 \%$. The overall seropositivity rate of HIV for 2009 - 2010 was $2.43 \%$ (Table 1). The total number of samples included in the study was 104. This selection was randomly done by including every fourth HIV positive serum sample, from all positive serum samples, for the years 2009 2010. Since the total number of HIV positive serum samples, for the 2009-2010 were 417, 104 serum samples got included in this study and formed the material for further testing (Table 2). The age and gender of patients whose serum samples were positive for HIV can be observed in (Table $3)$. Most patients were in the age group of 30 - 44 years $(54.8 \%)$ and 45 and above (26.9 $\%)$.

A low number of HIV seropositive individuals were in the age group of $15-29$ years and 0-14years (16.3\% and 1.92\%) respectively. Males were significantly more affected than females. The male to female ratio was 1.5:1. The male preponderance was observed in all age group except in age group 15-29 years where the females were more than males, although the difference was not statistically significant (males $=6$; Females $=11$ ). Sexual route formed the major mode of acquisition of HIV (73.07\%). 
Infection through infected needles and syringes constituted (15.38\%). Transmission of HIV from mother to child was seen in 3 patients $(2.88 \%)$. In 7 cases $(6.73 \%)$ the exact route of HIV acquisition could not be determined. Only 2 cases (1.92\%) were transmitted through blood and blood products (Table 4). Patients with various sexually transmitted diseases (STD) formed the major bulk of HIV positive persons $(50 \%)$. The individual breakup being, unknown cases $23.07 \%$, Commercial sex workers (CSW) $20.19 \%$. Individuals in the MSM (Men having sex with men) category were 3 in number (2.88\%). Seropositivity to the tune of $1.92 \%$ was seen in IV drug users \& Antenatal mothers (Table 5). 104 HIV positive serum samples which were included in the study were subjected to serological tests to determine the presence of markers for $\mathrm{HBV}, \mathrm{HCV}$, \& syphilis. VDRL test was positive in $6.7 \%$. Evidence of hepatitis B was observed in $4.8 \%(\mathrm{HBV}) \& 2 \% \mathrm{HCV}$ cases. Seven samples $(6.7 \%)$ were positive for hepatitis which was either HBV (4.8\%) or HCV $(1.9 \%)$. No samples were found to be positive for both HCV and HBV (Table 6). Table 7 compares the dual presence of viral hepatitis markers (HBV and / or HCV) and a positive VDRL test (indicator of syphilis) in HIV positive patients. Out of 104 HIV positive serum samples that formed the study material, 14 samples (13.4\%) were positive for markers of $\mathrm{HBV}$ or $\mathrm{HCV}$ or syphilis. No patients were positive for either of two markers, i.e. HBV + VDRL / HCV + VDRL or for all three markers, i.e., HBV, HCV and VDRL.

The purpose of present study was to find the Seroprevalence rate of syphilis, HBV and HCV coinfection in HIV positive patients. All the three STDS share common routes of transmission. Monitoring of HIV positive patients for co-infection with $\mathrm{HBV}$ and Hepatitis $\mathrm{C}$ is therefore necessary.

Table.1 Total number of blood samples tested for HIV

\begin{tabular}{|l|c|c|c|}
\hline Year & Total No. of Samples Tested & No Positive for HIV & Percentage Positive \\
\hline $\mathbf{2 0 0 9}$ & 11139 & 301 & 2.7 \\
\hline $\mathbf{2 0 1 0}$ & 5966 & 116 & 1.5 \\
\hline TOTAL & 17105 & 417 & 2.43 \\
\hline
\end{tabular}

Table.2 Number of HIV positive blood samples included in the study

\begin{tabular}{|l|c|c|}
\hline $\begin{array}{l}\text { Total no. of sera } \\
\text { tested }\end{array}$ & $\begin{array}{c}\text { Total no positive for } \\
\text { HIV }\end{array}$ & $\begin{array}{c}\text { No. included in the } \\
\text { study }\end{array}$ \\
\hline $\mathbf{1 7 1 0 5}$ & 417 & 104 \\
& $(2.43 \%)$ & $(24.94 \%)$ \\
\hline
\end{tabular}


Table.3 Age and gender wise distribution of HIV positive patients

\begin{tabular}{|l|c|c|c|}
\hline AGE & No. of Positive cases & Males & Females \\
\hline $\mathbf{0 - 1 4}$ & $2(1.92 \%)$ & $2(3.17)$ & $0(0)$ \\
\hline $\mathbf{1 5 - 2 9}$ & $17(16.3 \%)$ & $6(9.52)$ & $11(26.82)$ \\
\hline $\mathbf{3 0 - 4 4}$ & $57(54.8 \%)$ & $34(53.9)$ & $23(56.09)$ \\
\hline $\mathbf{4 5} \&$ above & $28(26.9 \%)$ & $21(33.33)$ & $7(17.07)$ \\
\hline Total & $104(24.94 \%)$ & $63(60.57)$ & $41(39.42)$. \\
\hline
\end{tabular}

Table.4 Route of transmission of HIV in positive cases

\begin{tabular}{|l|l|l|}
\hline Route of transmission & Positive cases & Percentages \\
\hline Sexual & 76 & 73.07 \\
\hline Infected needles \& syringes & 16 & 15.38 \\
\hline Blood \& blood products & 2 & 1.92 \\
\hline Parent to child transmission & 3 & 2.88 \\
\hline Unknown route & 7 & 6.73 \\
\hline TOTAL & 104 & 100 \\
\hline
\end{tabular}

Table.5 Categorical distribution of high risk groups

\begin{tabular}{|l|c|c|}
\hline GROUPS & Positive cases & Percentages \\
\hline STD & 52 & 50 \\
\hline CSW & 21 & 20.19 \\
\hline MSM & 3 & 2.88 \\
\hline IV DRUG USERS & 2 & 1.92 \\
\hline ANTENATAL & 2 & 1.92 \\
\hline LAB PERSONNEL & 0 & 0 \\
\hline UNKNOWN & 24 & 23.07 \\
\hline TOTAL & 104 & 100 \\
\hline
\end{tabular}

Table.6 Serological evidence of three STIs in 104 HIV positive individuals

\begin{tabular}{|l|l|l|l|}
\hline & HIV + HBV & HIV + HCV & HIV + VDRL \\
\hline Positive cases & 5 & 2 & 7 \\
\hline Percentages & $4.8 \%$ & $1.9 \%$ & $6.7 \%$ \\
\hline
\end{tabular}


Table.7 Association of VDRL positivity and hepatitis markers in HIV positive samples

\begin{tabular}{|l|l|l|l|l|l|l|l|}
\hline & $\begin{array}{l}\text { Total } \\
\text { no. of } \\
\text { samples }\end{array}$ & $\begin{array}{l}\text { HIV + HBV } \\
\text { or HCV or } \\
\text { VDRL }\end{array}$ & $\begin{array}{l}\text { HIV + } \\
\text { HBV or } \\
\text { HCV }\end{array}$ & $\begin{array}{l}\text { HBV+ } \\
\text { VDRL }\end{array}$ & $\begin{array}{l}\text { HCV+ } \\
\text { VDRL }\end{array}$ & $\begin{array}{l}\text { HBV+VDRL+ } \\
\text { HCV }\end{array}$ \\
\hline $\begin{array}{l}\text { Positive } \\
\text { cases }\end{array}$ & 104 & 14 & 7 & 0 & 0 & 0 \\
\hline Percentages & 100 & 13.4 & 6.7 & 0 & 0 & 0 \\
\hline
\end{tabular}

Table.8 Reports of Seroprevalence of co-infection with Hepatitis B, Hepatitis C and syphilis in HIV positive patients in India

\begin{tabular}{|c|c|c|c|c|c|c|}
\hline \multirow[t]{2}{*}{ S.No } & \multirow[t]{2}{*}{ Authors } & \multirow[t]{2}{*}{ Place of Study } & \multirow[t]{2}{*}{ Year } & \multicolumn{3}{|c|}{$\begin{array}{l}\text { Positivity rate in } \mathrm{HIV} \\
\text { patients }\end{array}$} \\
\hline & & & & VDRL & HBV & $\mathrm{HCV}$ \\
\hline 1 & U K Baveja etal & Delhi & 2003 & - & $14.72 \%$ & $9.64 \%$ \\
\hline 2 & SS Tanbhiwala etal & Nagpur & 2003 & - & $25.8 \%$ & $5.6 \%$ \\
\hline 3 & Swathi Gupta etal & New Delhi & 2006 & -- & $5.32 \%$ & $2.43 \%$ \\
\hline 4 & Saravanan et al & Chennai & 2007 & -- & $9 \%$ & $2.2 \%$ \\
\hline 5 & S U Munshi et al & Bangladesh & 2008 & $13.55 \%$ & $4.24 \%$ & $1.69 \%$ \\
\hline 6 & Present Study & Andhra Pradesh & 2010 & $6.7 \%$ & $4.8 \%$ & $1.9 \%$ \\
\hline 7 & SK Antala et al & Rajkot & 2010 & $6 \%$ & $19.5 \%$ & $3 \%$ \\
\hline 8 & Ashish chandraet al & Nepal & 2011 & - & - & $3.59 \%$ \\
\hline 9 & Sanjiv Ahuja et al & New Delhi & 2013 & - & $4.9 \%$ & $1.7 \%$ \\
\hline
\end{tabular}

Fig.1 Year wise distribution of HIV positivity rate.

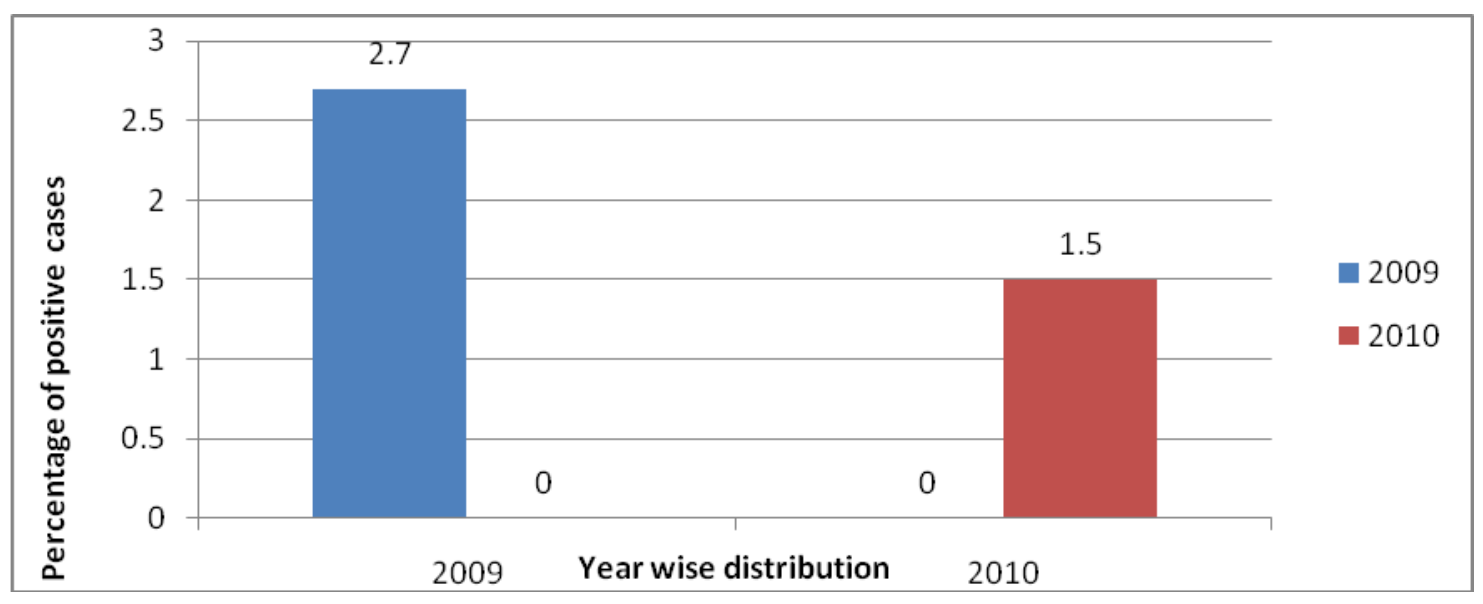


Fig.3 Age and gender wise distribution of HIV positive patients

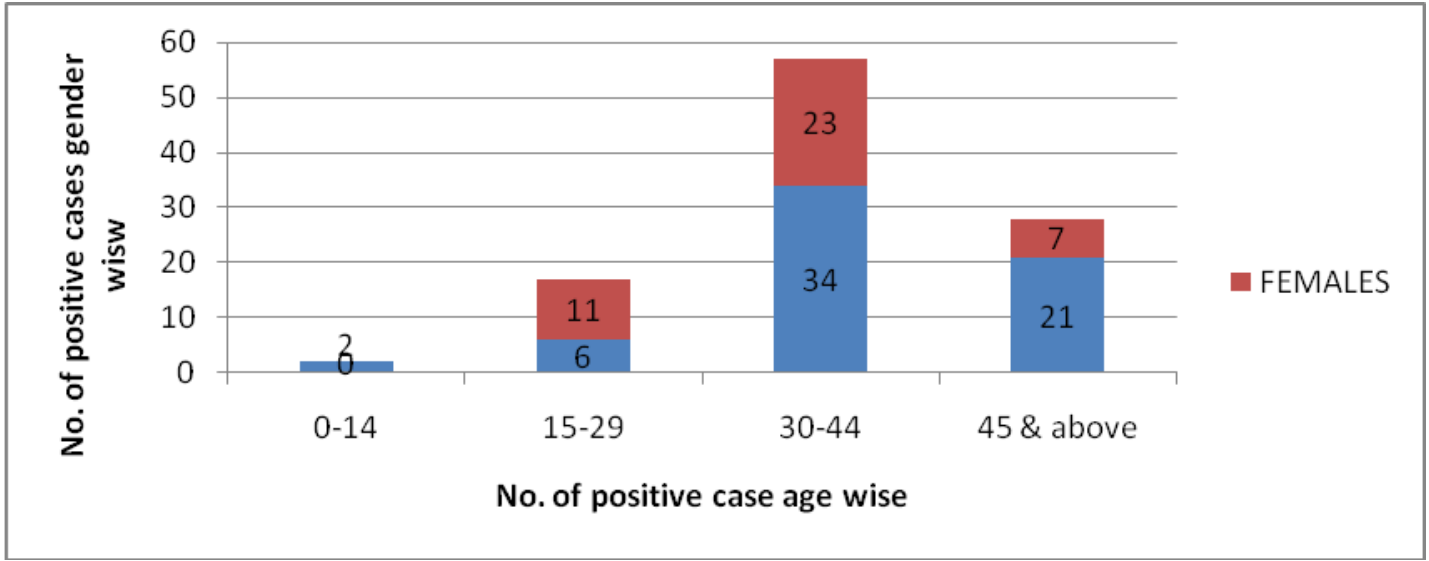

Fig.4 Route of transmission of HIV in positive cases

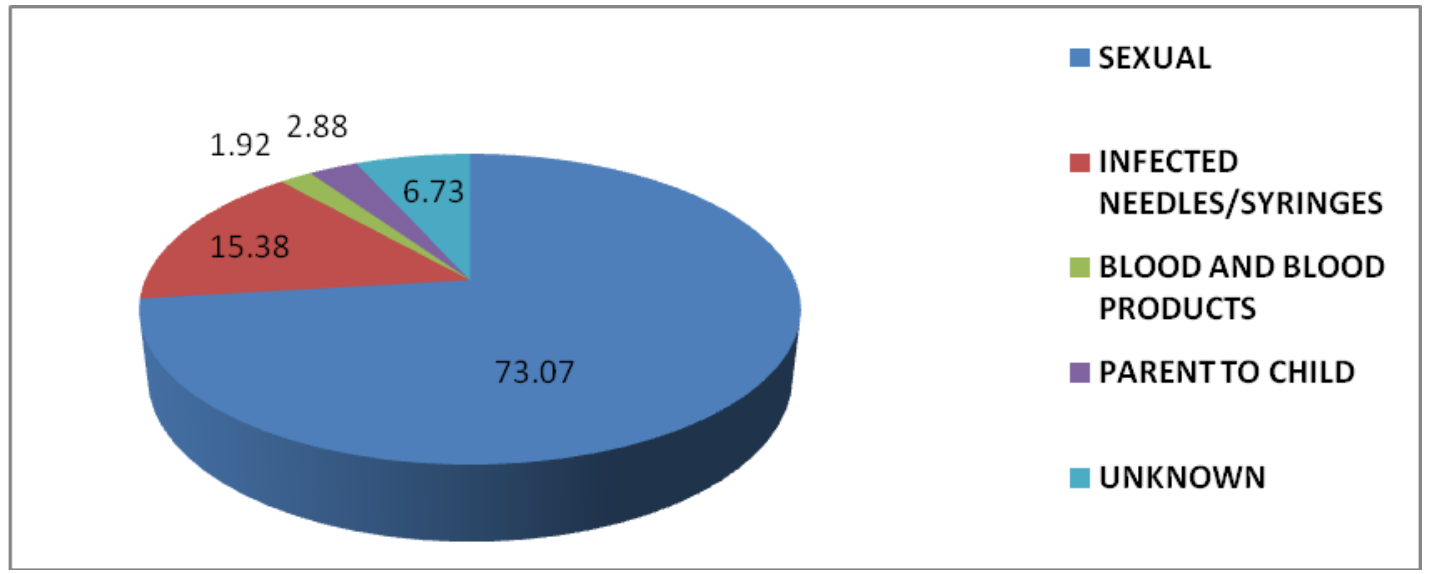

Fig.5 Categorical distribution of high risk groups

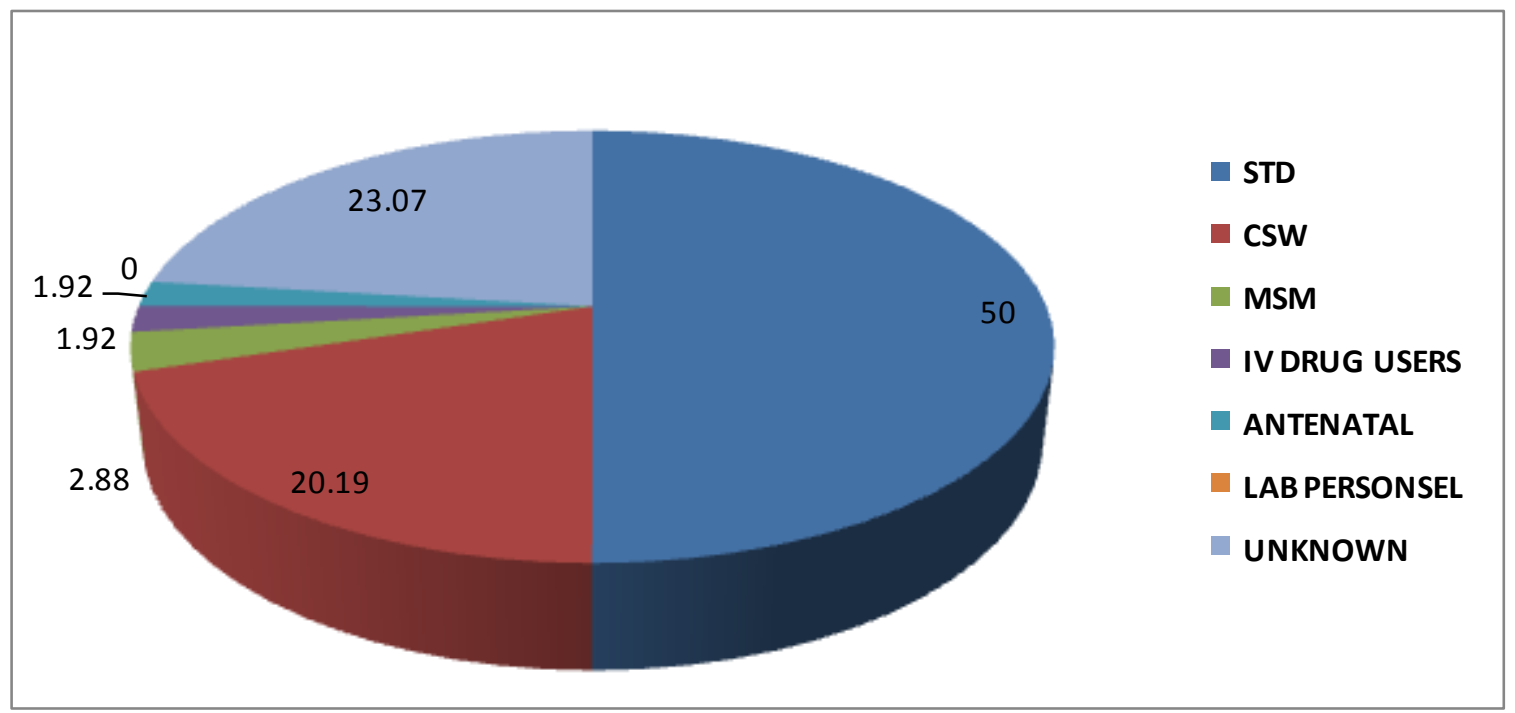


Fig.6 Serological evidence of three STIs in 104 HIV positive individuals

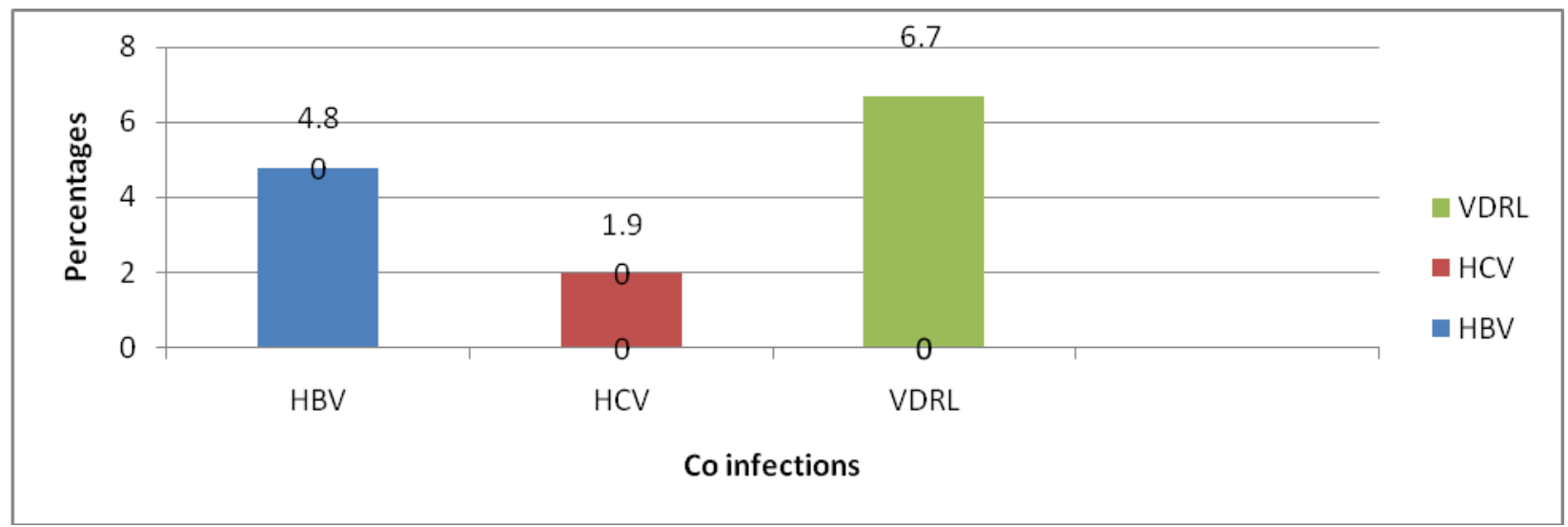

In our study HIV positivity rate in 2009 was $2.7 \%$ while in 2010 it was $1.5 \%$. Seropositivity rate for HIV was found to be 2.43. Among the HIV infected patients male predominance $(60.5 \%)$ was noted.. The mean age found to be $30-44$ yrs $(54.8 \%)$ which is similar to other studies (Shanmugam Saravanan et al., 2007). In a study conducted by Saravanan et al., 2007, in South India in HIV Positive individuals, sexual contact was most common route of transmission followed by unknown cause, blood transfusion and IDUs which is similar to our study. Heterosexual contact formed the major mode of transmission of HIV (73.07\%) in our study, followed by infected needles / syringes (15.38\%), Unknown cause $6.73 \%$, antenatal $2.88 \%$ and blood transfusion $1.92 \%$. Patients with various manifestations of sexually transmitted diseases like genital ulcers, either urethral discharge or urethral with cervical discharge and few with genital warts formed the major bulk of HIV positive patients (50\%) followed by CSW (20.19\%). In our study, the overall prevalence of co-infection in HIV patients was $13.4 \%$ and with hepatitis viruses alone it was $6.7 \%$. Triple infection was not detected in HIV positive patients in the present study. The rate of detection of VDRL positivity was higher (6.7\%) followed by $\mathrm{HbsAg}(4.8 \%)$ and $\mathrm{HCV}$
(1.9\%). In a study conducted by U K Baveja et al., 2003 in IDUs in Delhi, the prevalence of coinfection with HIV and HBV was found to be $14.72 \%$ and $\mathrm{HIV}$ and $\mathrm{HCV}$ 9.64\%, HIV with HBV, HCV and syphilis $1.52 \%$. Drug abuse is prevalent in metropolis and other cities. In our study only $2(1.92 \%)$ cases were IDUs, both were reactive for HIV only. Among the 7(6.7\%) of VDRL positive cases the route of transmission was sexual contact. In 5 (4.8\%) cases of HIV with HBV positivity, in 3 cases the route of transmission was sexual and in 2 cases blood transfusion. In 2 cases of HIV with HCV positivity the route of transmission was not known. None of the HIV positive cases were positive for all the three seromarkers. Seroprevalence of different seromarkers of hepatitis virus and VDRL positivity was compared with other studies Table 8 . We found that the seroprevalence of VDRL in HIV patients is higher $13.55 \%$ higher in a study conducted by SU Munshi et al., 2008, when compared to our study $6.7 \%$. Whereas HBV and HCV positivity in HIV patients is similar to our study. The HBV and HCV prevalence in our study group was lower than other studies from North India. This can be related to the type of risk groups in the other studies.

In conclusion, co-infection of HIV with 
syphilis, Hepatitis B and hepatitis $\mathrm{C}$ viruses is seen in $6.7 \%, 4.8 \%$ and $1.9 \%$ of patients, respectively. This suggest that apart from other opportunistic bacterial and fungal infections, HIV infected patients have high risk of getting Syphilis, $\mathrm{HBV} / \mathrm{HCV}$ infection due to enhanced immunodeficiency of HIV. The similar route of transmission also plays significant role and is of epidemiological importance in our country. Thus routine screening of HIV infected patients for co-infections with HBV/ HCV and syphilis should be made mandatory because these infections will increase the risk of cirrhosis, liver deficiency, chronic illness and mortalities in comparision to when a person is infected with only one of these viruses.

\section{Acknowledgements}

We sincerely thank our former Professor and HOD of Microbiology, Dr. K. Sree Rama Rao, for his valuable guidance without which the study could not have been completed. I also thank all the teaching and nonteaching staff of Department of Microbiology, NRI Medical College \& GH for their support.

\section{References}

Antala, S.K., et al. 2010. Seroprevalence of Hepatitis B, Hepatitis C and Syphilis in HIV positive cases at ICTC, Rajkot, Gujarat Med. J., vol.65.No.1

Ashish Chandra Shrestha et al. 2012. Coinfection rate of HIV, HBV and Syphilis among HCV seropositive identified blood donors in Kathmandu, Nepal. Infect. Ecol. Epidemiol., 2: vol 210.14835.

Baveja, U.K., D Chattopadhya et al. 2003. A cross sectional serological study of the coinfection of Hepatitis B virus, Hepatitis C virus and HIV amongst a cohort of IDUs at Delhi, Indian J. Med. Microbiol., 21(4): $280-283$.

Chung, R.T. 2006. Hepatitis C and B viruses the new oppurtunists in HIV infected. Top HIV Med., 14: 78-83

Krunal, D., et al. 2013. Seropositivity of Hepatitis B, Hepatitis C, Syphilis, and HIV in antenatal women in India. $J$. Infect. Dev. Ctries., 7(11): 832-837.

Munshi, S.U., et al. 2008. HBV,HCV and syphilis co-infection in Human immunodeficiency virus positive Bangladeshi patients: Observation at two reference laboratories, IJMM, vo.26,282283.1.

Sanjiv Ahuja et al. 2013. Seroprevalence of Hepatitis B and C Co-infection in HIV positive patients from a Tertiary Care Hospital, JIMSA, Vol.26 No.2

Shanmugam Saravanam, Vijayakumar Velu et al. 2007. Coinfection of hepatitis B and hepatitis $\mathrm{C}$ virus in HIV - infected patients in South India, World $J$. Gastroenterol., 13(37).

Swathi Gupta et al. 2006. Hepatitis B and C virus co-infection in Human immunodeficiency virus positive North Indian Patients. World J. Gastroenterol., 14: 12(42) 6879-6883.

Tankhiwale, S.S., R.K. Khadase, et al. 2003. Seroprevalence of Anti-HCV and Hepatitis B surface antigen in HIV infected patients. Indian J. Med. Microbiol., 21(4): 268-270.

\section{How to cite this article:}

Konda Palli Harathi Reddy, Uma Penmetcha, Ramesh Babu Myneni and Padmaja Yarlagadda. 2016. A Study of Seroprevalence of Hepatitis B, Hepatitis C and Syphilis Coinfection among HIV Patients in a Tertiary Care Teaching Hospital, South India. Int.J.Curr.Microbiol.App.Sci. 5(8): 699-707. doi: http://dx.doi.org/10.20546/ijcmas.2016.508.079 